\title{
International Conference on
}

\section{Sports Medicine and Fitness \\ March 23-25, 2015 Chicago, USA}

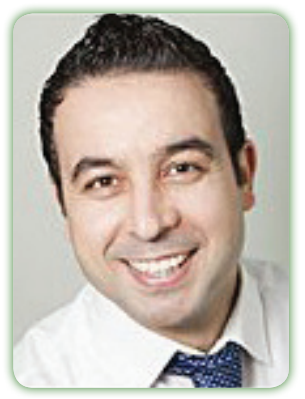

\section{Ahmad Alkhatib}

Qatar University, Qatar

\section{Efficacy of popular herbal supplements on fat metabolism and energy expenditure during exercise}

$\mathrm{N}$

either exercise alone nor diets alone are able to provide optimal weight loss and health outcomes mainly due to poor long-term compliance. Increasing energy expenditure is considered an essential factor for weight loss, reducing energy intake or absorption, and increase the exercise related effectiveness for weight loss and potentially several metabolic health outcomes, particularly when energy is expended from fat fuel energy sources. It is, therefore, unsurprising that popularity of several commercially available slimming aids, especially herbal plant supplements have been marketed for their efficacy, especially to increase EE and weight reduction. Consumption of these supplements has been reported in both obese and non-obese individuals who desire to lose weight, reduce or control body fat. Popular herbal plant supplements that have been reported in the scientific literature include caffeine and capsaicin supplements, Ayurvedic preparations and herbal tea consumption, resulting in various degrees of thermogenic effectiveness, especially in increasing resting energy expenditure and resting metabolic rate. With the exception of caffeine, limited research has investigated the exercise dependent EE and fat utilization during exercise, induced by these thermogenic supplements. During exercise fat metabolism increases several folds, and the least is known about the efficacy of herbal thermogenic aids at different exercise conditions. Recent research has shown promising effects of popular herbal teas such as Green tea, which contains several active polyphenolic catchins and Yerba Mate which is rich in caffeoyl derivatives, and both play an interesting role in elevating fatty acid oxidation and increasing energy expenditure at rest and during exercise.

\section{Biography}

Ahmad Alkhatib is the Director of Sport Science at Qatar University and an Associate Professor, Exercise Metabolism and has over16 years experience which included several leading academic positions including Suffolk, Lincoln, Greenwich and Sheffield-Hallam Universities. His PhD in Exercise Physiology and Metabolism and MSc in Sport Science (Health and Fitness) were awarded from the University of Essex, and he has over fifty research publications. His current research focuses on optimizing health and exercise performance, through novel nutritional and exercise interventions, particularly herbal supplements during exercise. He is a fully-registered Nutritionist (RNtur) and an accredited Sport Nutritionist (CISSN).

\section{Notes:}

